\title{
Development of Neonatal Respiratory and Intensive Care: Chinese Perspectives
}

\author{
Bo Sun ${ }^{a}$ b Li Ma $^{c}$ Xiaohong Liu ${ }^{d}$ Xirong Gao ${ }^{e}$ Liming $\mathrm{Ni}^{f}$ \\ ${ }^{a}$ Department of Neonatology, Children's Hospital of Fudan University, ${ }^{b}$ The Neonatal Laboratory, Ministry of \\ Health, Shanghai, Departments of Neonatology at ' Hebei Provincial Children's Hospital, Shijiazhuang, 'Shenzhen \\ Children's Hospital, Shenzhen, ${ }^{\mathrm{e} H u n a n}$ Provincial Children's Hospital, Changsha, and ${ }^{\mathrm{f}}$ Shaanxi Provincial Maternity \\ Hospital, Xi'an, China
}

\section{Key Words}

Neonatology $\cdot$ Prematurity $\cdot$ Respiratory distress syndrome $\cdot$ Respiratory failure $\cdot$ Respiratory therapy

\begin{abstract}
Recent economic improvements in China have allowed the development of perinatal-neonatal care in sub-provincial regions. However, variations in neonatal respiratory and intensive care exist, especially in regions with limited resources. We conducted a series of collaborative clinical investigations into neonatal hypoxemic respiratory failure (NRF). In the study period from 2004 to 2005, this nationwide study found an incidence of NRF of $13.4 \%$ of total admissions to neonatal intensive care units (NICUs), with a mortality of $32 \%$. Fewer than $30 \%$ of infants with respiratory distress syndrome (RDS) received surfactant treatment. Most cases of NRF had birth weights (BWs) of 1,000-1,500 g. Approximately $60 \%$ of deaths were due to withdrawal of respiratory support because of economic restraints despite initial response to therapy. Extremely low BW or gestational age accounted for less than $2 \%$ of all NRF cases, and their survival rate was less than $50 \%$. A prospective clinical epidemiologic study of NRF in 14 NICUs, mainly sub-provincial centers, in Hebei province was undertaken in the study period from 2007 to 2008. NRF made up $16.9 \%$ of total NICU admissions, with in-
\end{abstract}

creased use of surfactant $(>50 \%)$ and continuous positive airway pressure $(>80 \%)$ in this study. However, mortality due to RDS, meconium aspiration syndrome and pulmonary infection/sepsis remained higher than $30 \%$, in part affected by socioeconomic factors. With measures to assist hospitalized neonates from low income families in urban areas, as well as the 'new rural cooperative health care program' to subsidize families from rural areas, the quality and affordability of NICU services may be improved in the forthcoming years.

Copyright $\odot 2011$ S. Karger AG, Basel

\section{Background of the Perinatal-Neonatal Care System}

With the dramatic economic growth of the past decade, health care for women and children in China has emerged and developed to higher levels, as reflected by reductions in maternal deaths during childbirth to 48.5 per 100,000 and neonatal tetanus to 9 per 100,000 live births in 2006, and deaths of children under 5 years old to 20 per 1,000 in $2006-2007$, along with infant and neonatal deaths to $<15$ and $<10$ per 1,000 , respectively, with

This study was presented at the 25th International Workshop on Surfactant Replacement, Moscow (Russia), June 10-12, 2010.

\section{KARGER}

Fax +41613061234 E-Mail karger@karger.ch www.karger.com
Bo Sun, MD, PhD

Departments of Pediatrics and Neonatology

Children's Hospital of Fudan University

399 Wanyuan Road, Shanghai 201102 (China)

Tel. +86 216493 1217, E-Mail bsun@shmu.edu.cn 
regional variations of 3 - to 5 -fold between the urban and rural areas or between the coastal and remote regions, respectively. In the late 1990s, the leading causes of death in children under 5 years were prematurity and low birth weight (BW) and gestational age (GA), birth asphyxia, pneumonia, congenital heart diseases and asphyxia [1]. At that time, the average nationwide neonatal death rate was above 20 per 1,000 live births. Special neonatal care was mainly confined to major provincial hospitals, where the capacity and quality of neonatal intensive care units (NICUs) were low, qualified specialists were few and experience in respiratory support and intensive care was very limited. This was evidenced by a $40 \%$ death rate among term neonates with severe meconium aspiration syndrome (MAS) in children's hospitals in major cities [2]. For premature infants with respiratory distress syndrome (RDS), mortality in the whole country was probably above $50 \%$. Even in Beijing, surfactant treatment for RDS resulted in approximately $65 \%$ survival at 28 days of age, with rates of major complications ranging from $7-79 \%$ [3]. Nowadays, perinatal-neonatal care in coastal provinces and municipalities, such as Shanghai, Beijing, Tianjin, Guangdong, Zhejiang, Jiangsu and Fujian, is well advanced, whereas in most of the northern and mideastern provinces, standards of care are variable. In the northeastern, southwestern and northwestern provinces and autonomous regions, the quality of care is low, especially at county level. In all these regions, provincial and sub-provincial levels of care are mainly supported by local public health care systems and funds, and these regions are characterized by high birth rates and a large patient population with limited service capacity and quality as well as affordability.

Since 2005, the development of perinatal-neonatal medicine at sub-provincial tertiary centers (level III) has been advancing in the most populated provinces and regions in China, mainly in the mid-southern and mideastern regions, such as Hebei, Shandong, Henan, Hubei and Hunan provinces, each with populations ranging from 50-90 million. This was followed by provinces with relatively low economic development in the eastern, northern, northeastern and mid-western regions, such as Anhui, Jiangxi, Liaoning, Jilin, Guangxi, Shaanxi, Shanxi and Sichuan provinces. Many sub-provincial regional cities have established or are establishing perinatal-neonatal centers in either maternity and children's hospitals or regional general hospitals, each serving populations of 1-5 million, corresponding to 10,000-60,000 annual deliveries, through a network linking to 30-100 local hospitals (level I-II). Such organization has led to a steadily growing workload to meet the demand of special care, reduction in perinatal death rates, quality of survival and affordability for hospitalized newborns. In the study period from 2008 to 2009, a jointly funded government health care insurance system, termed the 'new rural cooperative health care program', was introduced [4]. This program covers $60-80 \%$ of the total health care expenditure, or up to 4-6 times the amount of the annual disposable income of most rural families [national average from 2008 to 2009 was CNY 4,500-5,000 (USD 650-700) equivalent per person], which allows rural families to obtain essential health care at very low cost. Implementation of this program may directly or indirectly affect the organization of perinatal-neonatal care in both rural and urban regions.

With urbanization and industrialization, more than a hundred million ( $8 \%$ of the total population of 1.3 billion by 2010) migrant workers from rural areas moved to Beijing, Shanghai and major cities in southeastern (coastal) provinces and regions to take part in construction and manufacturing. This contributed to $30-50 \%$ of admissions to local neonatal wards, many requiring respiratory support and intensive care. In recent years, measures were taken in these regions to enable migrant families to share essentially local perinatal-neonatal care. These include local funds to subsidize costs of hospital delivery and hospitalized neonatal care. Another solution was to cover the costs with the 'new rural cooperative health care program' [4] from their original regions of residence. In most neonatal special care services, there are hospital funds to meet unpaid hospital costs for those from very low income families.

In a retrospective survey, the Subspecialty Group of Neonatology of the Pediatric Society of the Chinese Medical Association [5] reported the clinical characteristics of 43,289 hospitalized neonates from 86 hospitals in 47 cities in 20 provinces during 12 consecutive months in 2005 . In these infants, the male-to-female ratio was $1.73: 1$ and the prematurity rate was $26.2 \%$, with $16.4 \%$ born late preterm (34-36 weeks' gestation). Major reasons for hospitalization were asphyxia, pneumonia and hypoxic-ischemic encephalopathy (HIE). Among those with respiratory disorders, $46.2 \%$ had pneumonia, $17.1 \%$ asphyxia, $3.6 \%$ MAS, 3.0\% apnea and only $2.5 \%$ RDS. HIE was found in $18.1 \%$ of the neonates, with mild, moderate and severe types accounting for 52,40 and $8 \%$, respectively. Overall, $63.9 \%$ of neonates recovered, $27.3 \%$ improved but did not recover, $7.6 \%$ requested own discharge (or withdrawal) and $1.2 \%$ were clinical deaths. Most deaths occurred in the first 3 days, with only $26.9 \%$ of deaths oc- 
curring after that period. The major causes of neonatal death were respiratory $(66.1 \%)$, cardiac $(12.2 \%)$ and renal (4.6\%) failure. Of the nonsurvivors, $49.2 \%$ had asphyxia, in contrast to $15.9 \%$ of the survivors. Maternal pregnancy-induced hypertension was significantly associated with neonatal asphyxia, small for GA, wet lung, RDS, pulmonary hemorrhage, MAS and HIE. Kang et al. [6], from Zhengzhou, Henan province, reported that the major causes of death in NICUs were RDS, sepsis and scleredema in preterm infants, whereas sepsis, asphyxia and pneumonia were commonest in term infants.

\section{A Nationwide Neonatal Network for Respiratory Diseases}

Between 2000 and 2003, most of the provincial perinatal-neonatal centers established NICUs with mechanical ventilation as a major means of life support. Surfactant treatment was also introduced for premature infants with RDS. Since then, we have conducted collaborative clinical investigations in three phases through a nationwide collaboration as a neonatal network for severe respiratory diseases, mainly targeted to neonatal hypoxemic respiratory failure (NRF) [7]. We speculated that, in resource-limited regions, it would be possible to improve the standard of NICUs by improving knowledge of clinical epidemiology and respiratory support. In the first phase, a multicenter randomized trial of surfactant $\left(\right.$ Curosurf ${ }^{\circledR}$ ) for treatment of MAS was undertaken in selected provincial NICUs [8]. The results revealed some efficacy of surfactant therapy and relatively low mortality $(<10 \%)$ for MAS. This study enabled specialists from major city NICUs to cooperate in solving critical care problems by adopting international criteria and methodology. In the second phase, from 2004 to 2005 , a prospective survey was undertaken in 23 NICUs at provincial level, 15 from southeastern (coastal) and 8 from mid-western areas [9]. This study showed that the incidence and mortality of NRF were $13.4 \%$ (of total admissions of critically ill neonates) and 32\%, respectively. Fewer than $30 \%$ of preterm babies with RDS received surfactant. The majority of problems in preterm neonates occurred in those with a BW of $1,000-1,500 \mathrm{~g}$ or a GA of $28-32$ weeks. About $60 \%$ of deaths were due to withdrawal from aggressive respiratory support. Neonates with an extremely low BW or GA accounted for less than $2 \%$ of this special NICU-based population. The mortality and cost of NRF differed significantly between NICUs in mid-western and southeastern regions, mainly associated with the or- ganization of perinatal care, NICU facility and staff competence, health insurance coverage and socioeconomic and cultural complexity [9-11]. In the third phase, during 2007-2010, we conducted several multicenter studies, either as a province-wide network or nationwide, employing interventional protocols for improvement of respiratory support and registration of NRF $[12,13]$.

\section{Neonatal Network in Hebei NICUs}

In 2007, we conducted a clinical epidemiologic study of NRF in 14 provincial and sub-provincial NICUs in Hebei province [12] using the same study protocol [9]. This province was in the mid-range of economic development, representative of ten provinces in northern and mid-eastern China. Prospectively collected data on neonatal NRF reflected approximately $75 \%$ of level III NICU capacity in the whole province, before the 'new rural cooperative health care program' was implemented (2008-2010). During the study period, neonatal in-hospital costs were almost fully borne by the parents.

For 11,100 NICU admissions during 12 consecutive months, the incidence of NRF was $16.9 \%(n=1,875)$, with more than $60 \%$ having a BW $<2,500 \mathrm{~g}$ and $2 \%<1,000 \mathrm{~g}$. More than $40 \%$ of NRF cases were due to RDS, and $58.3 \%$ received surfactant therapy. In those requiring respiratory support, continuous positive airway pressure (CPAP) was used more than conventional ventilation. The mortality due to NRF (31.4\%) was similar to that found in the nationwide study in the period from 2004 to 2005, and the mortality due to NRF with RDS, MAS and pneumonia was $30 \%$ or higher (table 1). Of nonsurvivors, 1 in 4 was reported as a 'failure of aggressive rescue', which means 3 in 4 deaths followed from the parental decision to withdraw care due to financial problems and/or concerns about poor prognosis. The mortality rate of NRF varied across the regions by up to 7-fold. The male-tofemale ratio of NRF was 2.6:1, and more male infants received surfactant than females [12]. Surfactant use in RDS in both studies achieved a nearly $15 \%$ net increment in survival (table 1), demonstrating its cost-effectiveness in these NICUs in the two study periods. The capacity and utilization of major respiratory therapies in the Hebei study tended to be the same as, or even better than, in the nationwide one, with similar outcome.

Moreover, analysis of the in-hospital data from the Hebei study showed the incidence of NRF to be 14.8 per 1,000 live births $(557 / 37,635)$, for which RDS, MAS and pneumonia/sepsis were diagnosed in $0.9,0.024$ and 
Table 1. Comparison of NRF data from nationwide and Hebei province network studies

\begin{tabular}{lcc}
\hline Variables & Nationwide & Hebei province \\
& Ref. No.: & [12]
\end{tabular}

Figures in parentheses represent percentages of NRF cases. ${ }^{1}$ Figures provided by personal communication.

\section{Related Studies in Guangdong}

In Shenzhen, Guangdong province, a neonatal network was established in 2006 aimed at raising the standard of respiratory support and intensive care and reducing NRF-related mortality. More than 18 neonatal units participated in the 12-month study, covering a total population of 12 million and 90,000 annual deliveries. Approximately $3 \%$ of admissions for neonatal special care suffered from NRF, of which about $30 \%$ were preterm. Mortality due to NRF was below $30 \%$, with RDS and MAS comprising 50 and $9 \%$, respectively. With an 'aggressive rescue effort', the survival rate of RDS and MAS in this survey was close to 80 and $70 \%$, respectively, reflecting the quality of respiratory support and intensive care. In this region, prophylactic surfactant was given to $13 \%$ of preterm infants below 30 weeks' gestation, and rescue surfactant was given to $75 \%$ of patients with RDS, the highest level in China. Han et al. [14] from the Guangdong Provincial Maternity Hospital reported that, between 2001 and 2006, the use of surfactant (Curosurf) significantly reduced the occurrence of RDS by $43-58 \%$ in those neonates less than 30 weeks of age and 30-31 weeks compared to nontreated controls. The study also revealed better outcome in the treatment group as regards 7-day mortality, length of ventilation, length of oxygen therapy and major complications. However, length of hospital stay was not significantly different.

\section{Estimation and Management of Premature Delivery}

Previously, another nationwide retrospective survey revealed a premature birth rate of about $8 \%$ in 80 urban hospitals [15]. Our recent study in an agricultural county (in Hebei province) with a population of more than 400,000 revealed a rate of $4.5 \%$ in a 12 -month period [16]. Our ongoing study in Huai'an (in Jiangsu province), with a population of 5 million, also revealed a premature delivery rate of $4 \%$ (unpublished data), suggesting a nationwide average rate of about $5-6 \%$ of all the deliveries by weighted sum in rural and urban populations, corresponding to $0.9-1$ million premature births. Our data on NRF in the Hebei NICU network was mainly in very low BW infants $(<1,500 \mathrm{~g})$ and term and near-term neonates. It is different from the data in North America and European countries, where in the late 1980s-1990s, care for very low $\mathrm{BW}$ and extremely low $\mathrm{BW}$ infants accounted for the major workload in NICUs [17-19], and since then early postnatal intervention for NRF and prevention of 
chronic lung disease (bronchopulmonary dysplasia) have been the major objectives. We anticipate that this will occur in the next decade in the Chinese urban neonatal population when perinatal-neonatal care will have fully developed and health insurance will cover much more than at present.

Cesarean section is now the major mode of delivery in many urban obstetric clinics, accounting for $50-70 \%$ of births in some areas or hospitals. The high cesarean section rate in urban hospitals results in substantial numbers of late preterm and term newborns with RDS, as reported in another retrospective survey by Chen et al. [20] from Quanzhou, Fujian province. In 196 out of 508 late preterm infants, 43 (26.5\%) developed RDS, 59 (30.1\%) transient tachypnea, 6 (3.1\%) MAS and 27 (13.7\%) pneumonia, whereas in term infants these incidences were 6.6, $14.5,15.2$ and $38.1 \%$, respectively. In late preterm infants with RDS, CPAP, conventional ventilation and surfactant were used in 26.5, 18.4 and $15.3 \%$ compared to $4.9,10.9$, and $3.6 \%$, respectively, in term infants. The overall survival rate in those infants with and without NRF was 91 and $95 \%$, respectively, along with incomplete (withdrawal from) treatment or death in 9 and 5\%, respectively. The cesarean section rate in an agricultural county hospital was around 30\% in the period between 2007 and 2008 [16], and RDS in late preterm and term infants was not so prominent. In a recent controlled multicenter study of inhaled nitric oxide therapy, mortality due to NRF was reduced to $11-15 \%$ in 200 term and near-term infants whose initial oxygenation index was $\geq 15$ and $70 \%$ of whom were delivered by cesarean section [13].

\section{Estimation of NICU Resources and Function}

The perinatal-neonatal care system in the north- and southwestern provinces/autonomous regions of China, such as Shaanxi, Ningxia, Gansu, Xinjiang, Qinghai, Tibet, Chongqing, Sichuan, Yunnan, Guizhou and Guangxi, regions which have lower gross domestic products, is under-developed with regard to both facilities and health care cost coverage. At the Hunan Provincial Children's Hospital, there were 33 physicians and 100 nurses for 200 beds in the neonatal department with 3 wards. Total admissions in 2009 were 5,778 , and about $25 \%$ required intensive care. There were 60 beds in the NICU, half for intensive and half for intermediate care, with 33 nurses and 16 physicians. In that province, this hospital serves as a main pediatric and neonatal emergency center with an extensive transport team. Nearly $50 \%$ of admissions were transferred from the regional hospital network through land transportation. For infants with a BW $>1,000$ or $<1,000 \mathrm{~g}$, the survival rate was 80 and $50 \%$, respectively. Respiratory support with mechanical ventilation was given to 650 infants, and the survival rate was $70 \%$ in those with BW $>1,000 \mathrm{~g}$, but in those with BW $<1,000 \mathrm{~g}$, survival was below $30 \%$. CPAP-treated infants had a $90 \%$ survival rate. In those treated with conventional and high-frequency ventilation, $70 \%$ recovered or were discharged, which was similar to the results of the nationwide and Hebei network studies $[9,12]$. This province is located in mid-southern China, with a population of 69 million and 780,000 births in 2009. This neonatal department ranks as one of the largest in capacity, representative of a provincial children's hospital-based NICU with relatively well-equipped facilities for respiratory support, and may be classified as IIIc equivalent according to North American standards [21]. In the whole province, there are about 40 level II and III hospitals with neonatal wards and 1,200 beds. NICUs are mainly located in the 22 hospitals in major provincial and sub-provincial cities, rarely in county hospitals.

A similar situation was encountered in the maternity hospitals of the northwestern provinces. In the Shaanxi Provincial Maternity Hospital, there were 11,000-12,000 births annually between 2008 and 2009, many of which were high-risk. The hospital received 1,800 cases into neonatal special care, with half transferred through the provincial network from 50 level I-II hospitals within a radius of $100 \mathrm{~km}$. The NICU was equipped with 4 conventional and 9 CPAP (Infant Flow System ${ }^{\circledR}$ ) ventilators, a bedside blood gas analyzer, X-ray and ultrasound apparatus. There were 50 beds, 40 nurses and 18 physicians (7 senior, 5 attending and 6 residents). This hospital, along with others in major cities of the northwestern provinces, constitutes the mainstay for regional perinatal-neonatal care. However, the standard of neonatal special care at sub-provincial tertiary centers is low. We speculate that, to ensure good quality and adequate neonatal special care in resource-limited regions, a target of 1 NICU bed for every 2,000 live births should at least be achieved. In other words, for every 100,000 live births, provision of 50 NICU beds (or 5 NICUs, each with 10 beds on average), mainly IIIa-IIIb [21], should be considered in sub-provincial regions, along with an appropriate standard of care commensurate with the best available domestic conditions.

In summary, a nationwide NICU network for improvement of neonatal respiratory care is feasible and would be advantageous. The clinical investigations in 
this patient population are relatively highly cost-effective based on daily service practice and relevant to the clinical situation where parents often have financial difficulties in maintaining 'rescue therapy'. The overall outcomes of NRF in both the nationwide and Hebei province studies show a clear trend of improvement in sub-provincial regional centers for neonatal special care. With further progress, this type of organization and experience should constitute a very important part of the neonatal community, linking developed and underdeveloped regions in China.

\section{Disclosure Statement}

This work was supported by grants from the '211 Project (Phase III)', Ministry of Education, China-Canada Joint Health Research Program (National Natural Science Foundation of China-Canadian Institutes of Health Research, No. 30611120518), and the Program for Outstanding Medical Science Leader (No. LJ06038), Shanghai Municipal Department of Health.

\section{References}

1 Wang YP, Miao L, Qian YQ, Liang J, Wu YQ, Zhu J, Dai L, Zhou GX: Analysis of under 5 years old children mortality and the leading death cause in China from 1996 to 2000 (in Chinese). Chin J Prev Med (Zhonghua Yu Fang Yi Xue Za Zhi) 2005;39:260-264.

2 Zhu JX, Zhou XD, Zhang XD, Sun MY, Zhang YM, Shen YH, Song SM, Chen KZ, Li $\mathrm{KH}$, Song GW, Sun B: Analysis of clinical management and death risks of severe meconium aspiration syndrome (in Chinese). Chin J Prac Pediatr (Zhong Guo Shi Yong Er Ke Za Zhi) 2001;16:277-279.

3 Li J, Fan XM, Song GW: A multicenter clinical trial of the surfactant replacement therapy for neonatal respiratory distress syndrome (in Chinese). Chin J Pediatr (Zhong Hua Er Ke Za Zhi) 2000;38:344-346.

4 Health Department of Hebei Province: The new rural cooperative medical care in central and western regions management capacity-building project management in 2009 (in Chinese). http://www.hebwst.gov.cn.

5 Subspecialty Group of Neonatology, Pediatric Society, Chinese Medical Association: Epidemiologic survey for hospitalized neonates in China (in Chinese). Chin J Contemp Pediatr (Zhong Guo Dang Dai Er Ke Za Zhi) 2009;11:15-20.

6 Kang WQ, Liu DP, Chen YH, Sun HQ, Xiong $\mathrm{H}$ : Analysis of neonatal death in NICU during the latest 5 years (in Chinese). Chin Pediatr Emerg Med (Zhong Guo Xiao Er Ji Jiu Yi Xue) 2009;16:454-455.

-7 Sun B, Qian LL, Liu CQ, Wang SN, Yu JL, Cheng XY: Development of perinatal-neonatal medicine in China. Neoreviews 2008; 9:e95-e101.

$\checkmark 8$ Chinese Collaborative Study Group for Neonatal Respiratory Diseases: Treatment of severe meconium aspiration syndrome with porcine surfactant: a multicenter, randomized, controlled trial. Acta Paediatr 2005;94: 896-902.
9 Qian L, Liu C, Zhuang W, Guo Y, Yu J, Chen $\mathrm{H}$, Wang S, Lin Z, Xia S, Ni L, Liu X, Chen C, Sun B; Chinese Collaborative Study Group for Neonatal Respiratory Diseases: Neonatal respiratory failure: a 12 -month clinical epidemiologic study from 2004 to 2005 in China. Pediatrics 2008;121:e1115-e1124

10 Chinese Collaborative Study Group for Neonatal Respiratory Diseases: Regional variation in practice and outcome of neonatal respiratory distress syndrome between the South-east and Mid-western China (in Chinese). Chin J Perinat Med (Zhong Hua Wei Chan Yi Xue Za Zhi) 2009;12:121-126.

-11 Qian LL, Liu CQ, Guo YX, Jiang YJ, Ni LM, Xia SW, Liu XH, Zhuang WZ, Xiao ZH, Wang SN, Zhou XY, Sun B; Chinese Collaborative Study Group for Neonatal Respiratory Diseases: Current status of neonatal acute respiratory disorders: a one-year prospective survey from a Chinese neonatal network. Chin Med J 2010;123:2769-2775.

$>12$ Ma L, Liu C, Wang Y, Li S, Zhai S, Gu X, Liu F, Yan A, Guo W, Li Y, Xiao M, Yin J, Li Y, Liu X, Wang R, Kirpalani H, Sun B: Mortality of neonatal respiratory failure related to socioeconomic factors in Hebei province of China. Neonatology 2010;100:14-22.

13 Wang YF, Liu CQ, Gao XR, Yang CY, Shan RB, Zhuang DY, Chen DM, Ni LM, Wang H, Xia SW, Chen C, Sun B and Collaborative Study Group for Neonatal Respiratory Diseases: Effects of inhaled nitric oxide in neonatal hypoxemic respiratory failure in resource limited condition. Chin Med J 2011; 124:1156-1163.
14 Han QH, Nie C, Wang B, Luo XQ, Wu X, He WY, Chen WC, Liu GS: The prophylaxis of pulmonary surfactant on hyaline membrane disease in preterm infants with different gestational age (in Chinese). Chin Pediatr Emerg Med (Zhong Guo Xiao Er Ji Jiu Yi Xue) 2009;17:123-126.

15 The Subspecialty Group of Neonatology, Chinese Pediatric Society: An initial epidemiologic investigation of preterm infants in cities of China (in Chinese). Chin Contemp J Pediatr 2005;7:25-28.

$\checkmark 16$ Ma L, Liu CQ, Zheng XL, Xin SF, Ji ZL, Li YM, Yan GP, Tian CF, Tang LM, Sun B: A 12-month prospective survey of perinatal outcome of liveborn neonates in Julu county, Hebei. Chin Med J 2010;123:2781-2785.

- 17 Horbar JD, Badger GJ, Carpenter JH, Fanaroff AA, Kilpatrick S, LaCorte M, Phibbs R, Soll RF; Members of the Vermont Oxford Network: Trends in mortality and morbidity for very low birth weight infants, 1991-1999. Pediatrics 2002;110:143-151.

18 Markestad T, Kaaresen PI, Rønnestad A, Reigstad H, Lossius K, Medbø S, Zanussi G, Engelund IE, Skjaerven R, Irgens LM; Norwegian Extreme Prematurity Study Group: Early death, morbidity, and need of treatment among extremely premature infants. Pediatrics 2005;115:1289-1298.

19 Angus DC, Linde-Zwirble WT, Clermont G, Griffin MF, Clark RH: Epidemiology of neonatal respiratory failure in the United States: projections from California and New York. Am J Respir Crit Care Med 2001;164:11541160 .

20 Chen DM, He Y, Wang RQ: Retrospective survey on health issues of later preterm infants (in Chinese). Chin Pediatr Emerg Med 2009;16:456-457.

21 Stark AR; American Academy of Pediatrics Committee on Fetus and Newborn: Levels of neonatal care. Pediatrics 2004;114:13411347. 